Published in final edited form as:

Mol Psychiatry. 2016 February ; 21(2): 178-188. doi:10.1038/mp.2015.128.

\title{
Altered neuronal network and rescue in a human MECP2 duplication model
}

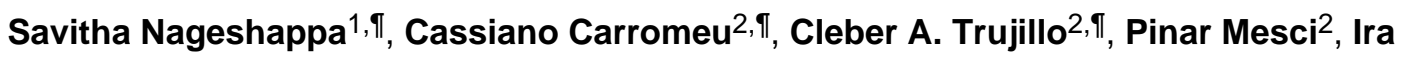 \\ Espuny-Camacho ${ }^{3,4}$, Emanuela Pasciuto ${ }^{4,5}$, Pierre Vanderhaeghen ${ }^{3,4,6}$, Catherine \\ Verfaillie $^{7}$, Susanna Raitano ${ }^{7}$, Anujith Kumar ${ }^{7,8}$, Claudia M.B. Carvalho ${ }^{9,10}$, Claudia \\ Bagni $^{4,5,11}$, Melissa B. Ramocki ${ }^{12}$, Bruno H. S. Araujo ${ }^{2}$, Laila B. Torres ${ }^{2}$, James R. Lupski ${ }^{9}$,

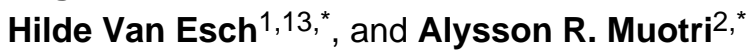 \\ ${ }^{1}$ Laboratory for the Genetics of Cognition, Center for Human Genetics, KU Leuven, Herestraat \\ 49, 3000 Leuven, Belgium \\ ${ }^{2}$ University of California San Diego, School of Medicine, Department of Pediatrics/Rady \\ Children's Hospital San Diego, Department of Cellular \& Molecular Medicine, Stem Cell Program, \\ La Jolla, CA 92093, MC 0695, USA
}

${ }^{3}$ Institut de Recherches en Biologie Humaine et Moléculaire (IRIBHM), Université Libre de Bruxelles (U.L.B.), Brussels, Belgium

${ }^{4} \mathrm{VIB}$ Center for the Biology of Disease, Leuven, Belgium

${ }^{5} \mathrm{KU}$ Leuven Center for Human Genetics and Leuven Institute for Neurodegenerative Diseases (LIND), KULeuven, Belgium

${ }^{6}$ WELBIO, B-1070 Brussels, Belgium

${ }^{7}$ Stem Cell Institute Leuven, Department of Development and Regeneration, Cluster Stem Cell Biology and Embryology, KU Leuven Medical School, Leuven, Belgium

${ }^{8}$ Manipal Institute of Regenerative Medicine, Yelahanka-560065, Bangalore, India

${ }^{9}$ Department of Molecular and Human Genetics, and Human Genome Sequencing Center Baylor College of Medicine, Houston, TX 77030, USA

${ }^{10}$ Centro de Pesquisas René Rachou - FIOCRUZ, Belo Horizonte, MG, Brazil

${ }^{11}$ University of Rome Tor Vergata, Department of Biomedicine and Prevention, Rome, Italy

${ }^{12}$ Department of Pediatrics, Section of Pediatric Neurology and Developmental Neuroscience, Baylor College of Medicine, Houston, TX 77030, USA

${ }^{13}$ Department of Clinical genetics, Center for Human Genetics, University Hospitals Leuven, Leuven, Belgium

\footnotetext{
Users may view, print, copy, and download text and data-mine the content in such documents, for the purposes of academic research, subject always to the full Conditions of use:http://www.nature.com/authors/editorial_policies/license.html\#terms

${ }^{*}$ Corresponding authors: Prof. Dr. Alysson R. Muotri (muotri@ucsd.edu). Prof. Dr. Hilde Van Esch (hilde.vanesch@uzleuven.be).

IThe authors contributed equally to the work.

The authors declare no conflict of interest.

Supplementary information is available at the Molecular Psychiatry website.
} 


\section{Abstract}

Increased dosage of $\mathrm{MeCP} 2$ results in a dramatic neurodevelopmental phenotype with onset at birth. We generated induced pluripotent stem cells (iPSC) from patients with the MECP2 duplication syndrome (MECP2dup), carrying different duplication sizes, to study the impact of increased MeCP2 dosage in human neurons. We show that cortical neurons derived from these different MECP2dup iPSC lines have increase synaptogenesis and dendritic complexity. Additionally, using multi-electrodes arrays, we show that neuronal network synchronization was altered in MECP2dup-derived neurons. Given MeCP2 function at the epigenetic level, we tested if these alterations were reversible using a library of compounds with defined activity on epigenetic pathways. One histone deacetylase inhibitor, $\mathrm{NCH}-51$, was validated as a potential clinical candidate. Interestingly, this compound has never been considered before as a therapeutic alternative for neurological disorders. Our model recapitulates early stages of the human MECP2 duplication syndrome and represents a promising cellular tool to facilitate therapeutic drug screening for severe neurodevelopmental disorders.

\section{Keywords}

$\mathrm{MeCP} 2$; duplication syndrome; induced pluripotent stem cells; disease modeling; epigenetic drugs; HDAC inhibitors; NCH-51; drug screening; multi-electrode arrays

\section{Introduction}

We previously showed that increased dosage of methyl-CpG-binding protein-2 (MeCP2) leads to a severe neurodevelopmental disorder in males, designated as the MECP2 duplication syndrome (MECP2dup) (MIM\#300260) ${ }^{1,2}$. The increased dosage of MeCP2 is the result of a copy number gain at Xq28, including the $M E C P 2$ gene, and results in severe to profound neurodevelopmental delay with onset at birth, limited or absent speech, hypotonia, epilepsy, autistic behavior and motor dysfunction ${ }^{1-5}$. Several studies in transgenic mice, overexpressing $M E C P 2$, have shown a progressive neurological phenotype, including tremor, gait ataxia, seizures, anxiety, abnormal learning and behavior and early death, recapitulating some aspects of the human disease ${ }^{6-9}$. The MeCP2 protein is widely expressed in various human tissues, but its increasing expression in the postnatal neural lineage suggests a key role in neurodevelopment, including the maturation, dendritic arborization, and axonal outgrowth of central nervous system ${ }^{10,11}$. Loss-of-function mutations in MECP2 cause Rett syndrome (RTT) (MIM\#312750) as well as other neurobehavioral disorders, indicating that $M E C P 2$ gene dosage is critical for human neurodevelopment ${ }^{12}$.

Human induced pluripotent stem cells (iPSCs) constitute a promising tool for investigating the underlying pathophysiology of traditionally challenging neurodevelopmental disorders ${ }^{13}$. We have demonstrated the utility of iPSCs to investigate the functional consequences of MECP2 loss of function ${ }^{14,15}$. Neurons generated from RTT patients' iPSCs exhibited several alterations, including decreased soma size, altered dendritic spine density and reduced excitatory synapses. Therefore, functional studies using iPSC-derived neuronal cultures from patients carrying $M E C P 2$ mutations can help delineate the mechanism by 
which alterations in $\mathrm{MeCP} 2$ dosage lead to disease and, ultimately, serve as a screening system to test potential therapeutic compounds. In this study, we present the development and analysis of a human iPSC model for the MECP2 duplication syndrome. In addition, we screened an epigenetic chemical library and found one compound able to successfully reverse several aspects of the observed neuronal phenotype.

\section{Materials and Methods}

\section{Patient consent}

This project was approved by the Ethics Committee of the Institutes where the study was conducted. After a complete description of the study, written informed consent was signed by the parents of the patients involved.

\section{Patient ascertainment}

Fibroblasts were obtained from three independent patients diagnosed with $M E C P 2$ duplication syndrome:

Patient 1 (male) was born after an uneventful pregnancy as the first child to healthy nonconsanguineous parents. At birth, he was very hypotonic with feeding problems. His development was severely delayed, with sitting at the age of 2 years and walking with support at the age of 3 years and half. He did not develop any active speech and shows repetitive behavior and hand flapping when he is excited. He suffered from recurrent infections occurring since the first months of life, necessitating almost continuous antibiotic therapy and frequent hospitalizations. At the age of 6 years, he was ventilated for 1 week because of a serious pneumonia. After this period, he lost ambulation and his epilepsy got worse. Array comparative genome hybridisation (array-CGH) at the age of 2 years showed a very small duplication of $300 \mathrm{~kb}$ at Xq28 (152.73-153.02 Mb). Within this interval, MECP2 is the only known brain expressed gene involved in a human disorder (Figure 1a).

Patient 2 (male) was born from non-consanguineous parents with negative family history. He showed severe hypotonia at birth and was severely delayed. Feeding and gross motor movements were always with support. The patient is non-verbal with severe stereotypic behaviour, including hand flapping. He was frequently hospitalized because of recurrent lung infections and seizures. Array-CGH revealed that the patient has a large duplication of 15.25 Mb at Xq28 (139.33-154.58 Mb) that includes MECP2 (Figure 1a).

Patient 3 (male) was born at term as the first child of healthy non-consanguineous parents. His family history is significant for three maternal uncles and a maternal cousin who died in the second or third decades of life with a similar clinical picture. The patient had developmental delays from birth. He had severe gastroesophageal reflux and was difficult to soothe as an infant. He sat up at one year, did not crawl, and did not walk until 2.5 years. He exhibited language delays from birth and learned some basic communication skills using a PECS board or basic signs. Hand and mouth stereotypies were frequent. He was formally diagnosed with autism at age 7 years. Medically, he experienced constipation, drooling, bruxism, and recurrent respiratory infections. At age 8 years, he developed medically refractory epilepsy and experienced developmental regression. He lost the ability to 
ambulate and became wheelchair dependent. He lost use of his hands and the limited interactive skills that he had gained. Severe dysphagia developed, and a gastrostomy tube was placed and is his sole source of nutrition. Seizures persist despite placement of a vagus nerve stimulator and use of the ketogenic diet. Pneumonias became frequent despite the use of prophylactic antibiotics and necessitated hospitalization and ventilatory support on numerous occasions. Array-CGH revealed that the patient has duplication of $500 \mathrm{~kb}$ at $\mathrm{Xq} 28$ (152.66-153.15 Mb) that includes MECP2 (Figure 1a). Patient 3 was previously described in Carvalho et al., 2013 (as patient BAB2623) ${ }^{18}$.

\section{Cell culture}

Inactivated mouse embryonic fibroblasts (iMEFs, GlobalStem) were maintained in DMEM HG (Life Technologies, CA, USA) containing 10\% FBS (Sigma-Aldrich, St Louis, MO, USA). Skin fibroblasts obtained from explants of dermal biopsy of male patients with $M E C P 2$ duplication syndrome and BJ1 fibroblast cells were maintained in DMEM F-12 (Life Technologies) containing 10\% FBS. iPSC clones were normally expanded on iMEF feeders using hES medium comprising DMEM-F12, 10\% KSR (both from Life Technologies), 200mM L-Glutamine, beta-mercaptoethanol (both from Sigma-Aldrich) and basic FGF (Life Technologies) and passaged by using collagenase. For feeder-free culturing, cells were grown on matrigel coated dishes (BD Bioscience, San Jose, CA, USA) and mTeSR1 from StemCell Technologies and passaged by using dispase.

\section{Generation of iPSCs}

Skin fibroblasts derived from patients with $M E C P 2$ duplication syndrome were infected with pMXs retroviral vectors containing coding sequences of human OCT4, SOX2, KLF4 and $C$-MYC (obtained from Addgene, Cambridge, MA, USA) ${ }^{19}$. Four days post infection, fibroblasts were trypsinized to singles and plated on the iMEF feeders and cultured using hESC medium. After 3-4 weeks, iPSC clones were manually picked and were further propagated clonally on feeders. Control 1 was derived from normal, healthy male donor fibroblasts (kind gift of Prof. Nadif-Kasri N., Nijmegen, The Netherlands). Control 2 was also generated from healthy male donor fibroblasts (Muotri lab, UCSD). The Control 3 iPSC clone was derived from BJ1 fibroblasts (Verfaillie lab, KULeuven).

\section{RNA extraction and RT-qPCR}

Total RNA was extracted using RNeasy Micro Kit from Qiagen. cDNA was synthesized by taking $1 \mu \mathrm{g}$ of total RNA and superscript III first-strand kit (Life Technologies) as per manufacturer's instruction. RT-qPCR was performed using gene specific primers (primer sequences available on request) and SYBR Green Platinum SuperMix from (Life Technologies). The expression of target genes and the endogenous control were measured with technical duplicates in each qRT-PCR reaction. To obtain statistical significance, values from a minimum of three independent differentiation qRT-PCR runs were considered. GAPDH was used as the endogenous control. The cycle threshold number $\left(\mathrm{C}_{\mathrm{t}}\right)$ was calculated using RQ Manager 1.2 Software (Life Technologies). The relative expressions of each target gene across differentiation days were normalized using $2^{(-\Delta \Delta C t)}$ method compared to differentiation day 5. Similarly, the fold expression of genes in the 
patient differentiation samples were calculated using $2^{(-\Delta \Delta C t)}$ method compared to differentiation day 5 of control cells.

\section{Immunocytochemistry}

Cells grown on coverslips were fixed using 4\% PFA for 20 minutes at room temperature (RT). Cells were permeabilized and blocked using a buffer containing 5\% Horse/Donkey/ Goat serum, $3 \%$ BSA and $0.3 \%$ TritonX-100 for 30 minutes at RT. Primary antibodies diluted in antibody diluent ( $1 \%$ Horse/Donkey/Goat serum $+3 \%$ BSA $+0.1 \%$ TritonX-100) were incubated overnight at $4{ }^{\circ} \mathrm{C}$. Secondary antibodies were incubated for $1 \mathrm{~h}$ at RT. Cells were washed three times with PBS $+0.1 \%$ Tween-20. Hoechst $(4 \mu \mathrm{g} / \mathrm{mL})$ was used to visualize nuclei. Coverslips were mounted on slides using Prolong Gold mounting medium (Life Technologies). Images were acquired using confocal microscope Radiance 2100 (Zeiss, Germany) equipped with an upright microscope (Eclipse E800; Nikon, Japan). The Supplementary Table 5 brings a detailed description of the antibodies used in this study.

\section{Teratoma formation}

Fully-grown iPSCs on feeders were dissociated by using collagenase for 3-4 minutes at $37^{\circ} \mathrm{C}$. Cells re-suspended in PBS and matrigel (1:1) were injected subcutaneously into dorsal flanks of immune-compromised Rag2-/- GammaC-/- mice. Five to six weeks later, teratomas were excised and fixed by using $4 \%$ PFA at $4{ }^{\circ} \mathrm{C}$ for overnight. Fixed tumor was embedded in paraffin and sections of $5 \mu \mathrm{m}$ thickness were made using microtome for histological studies. The tissue sections were stained with haematoxylin \& eosin (H\&E) and analyzed for the presence of the different germ layer tissues.

\section{EB formation}

Feeder free cultured iPSCs were dissociated using dispase for 5 minutes at $37^{\circ} \mathrm{C}$ and centrifuged for 3 minutes at $1200 \mathrm{rpm}$. Cell pellet was re-suspended in EB medium [IMDM medium, 15\% FBS, 2 mM L-glutamine, $1 \%$ non-essential amino acids, $1 \mathrm{mM}$ Sodium pyruvate, $100 \mathrm{U}$ penicillin/streptomycin, $200 \mu \mathrm{g} / \mathrm{ml}$ Iron-saturated-transferrin, $10 \mu \mathrm{M} \beta$ mercaptoethanol, $50 \mu \mathrm{g} / \mathrm{mL}$ ascorbic acid (Sigma-Aldrich)] supplemented with ROCK inhibitor and plated on 'low attachment plate'. Medium was changed every alternate day by centrifuging at $700 \mathrm{rpm}$ for 1 minute and re-suspending gently using fresh medium. After 8 days, EBs were re-plated on a plate pre-coated with gelatin for 30 minutes at RT. The attached cells were maintained for 8 more days in EB medium and were taken for gene expression analysis.

\section{Protein isolation and Immunoblotting}

Cells were harvested and lysed in a buffer containing 50mM Tris- $\mathrm{HCl}$ at $\mathrm{pH} 7.4,300 \mathrm{mM}$ $\mathrm{NaCl}, 1 \mathrm{mM}$ DTT, $0.5 \%$ TritonX-100, protease inhibitors and sonicated briefly. Total cell lysates were centrifuged at $6000 \mathrm{rpm}$ for 10 minutes at $4^{\circ} \mathrm{C}$. Protein concentration was measured using Bradford's reagent. Subsequently, $30 \mu \mathrm{g}$ of proteins were loaded on NuPAGE, 10\% Bis-Tris Gel using MOPS buffer at $200 \mathrm{~V}$ for $1 \mathrm{~h}$ and then transferred to PVDF membrane using Life Technologies iBlot system. Further, membrane blocking was performed using TBS with 5\% milk powder and $0.1 \%$ Tween-20 for 45 minutes at RT. The 
membrane was incubated with primary antibody, mouse anti-MeCP2 (1:1000; SigmaAldrich) diluted in blocking buffer for overnight at $4^{\circ} \mathrm{C}$. HRP-conjugated anti-mouse antibody was used as secondary and incubated for $1 \mathrm{~h}$ at RT. Rabbit anti-GAPDH was used as a loading control. Proteins were detected using Western Blot Chemiluminescence reagent Plus kit (Perkin-Elmer, Waltham, MA, USA) as per manufacturer's instruction.

\section{Chromatin Immunoprecipitation assay}

ChIP assay was performed using the ChIP-IT Express Enzymatic kit (Active Motif, Carlsbad, CA, USA). Briefly, starting with $10 \mathrm{~cm}$ plates, cross-link was induced in cells with $1 \%$ formaldehyde for $10 \mathrm{~min}$ at RT. After washout with ice-cold PBS, the reaction was stopped with Glycine Stop-Fix Solution for $5 \mathrm{~min}$ and plates were washed again with PBS. Cells were then suspended in Cell Scraping Solution and pelleted by centrifugation $(720 \times \mathrm{g}$ for $10 \mathrm{~min}$ at $4^{\circ} \mathrm{C}$ ). The cell pellet was re-suspended in $1 \mathrm{~mL}$ of ice-cold Lysis Buffer, Dounce homogenized with 15 strokes and centrifuged for $10 \mathrm{~min}$ at $2400 \times \mathrm{g}$ and $4^{\circ} \mathrm{C}$. The nuclei pellet was re-suspended in $350 \mu \mathrm{L}$ of Digestion Buffer. After 5 min incubation at $37^{\circ} \mathrm{C}$, the solution was supplemented with $17 \mu \mathrm{L}$ of Enzymatic Shearing Cocktail (200 $\mathrm{U} / \mathrm{mL}$ ) and incubated for more $15 \mathrm{~min}$ at $37^{\circ} \mathrm{C}$. The ChIP reaction was setup with $25 \mu \mathrm{g}$ of sheared chromatin, $2 \mu \mathrm{g}$ of anti-MeCP2 antibody (Diagenode, Denville, NJ, USA) and Protein G Magnetic Beads. The reaction was incubated on an end-to-end rotator for $16 \mathrm{~h}$ at $4^{\circ} \mathrm{C}$. A magnetic stand was used collect the magnetic beads at the end of incubation and subsequent washes (once with ChIP Buffer 1 and twice with ChIP Buffer 2). Washed beads were re-suspended in $50 \mu \mathrm{L}$ of Elution Buffer AM2 and incubate at room temperature under agitation for $15 \mathrm{~min}$. Cross-link was reverted with $50 \mu \mathrm{L}$ of Reverse Cross-linking Buffer, removal of supernatant after pelleting the beads with the magnetic stand and incubation of the solution at $95^{\circ} \mathrm{C}$ for $15 \mathrm{~min}$. Finally, the supernatant was incubated at $37^{\circ} \mathrm{C}$ for $1 \mathrm{~h}$ with Proteinase K followed by addition of $2 \mu \mathrm{L}$ of Proteinase K Stop Solution. The DNA was immediately used on endpoint PCR and quantitative PCR reactions (primer sequences available on request). The antibody conditions for the ChIP assay were validated with brainderived neurotrophic factor (BDNF) promoter in exon IV and another region (negative) of the promoter as previously described ${ }^{20}$.

\section{Copy number variation (CNV) analysis by array Comparative Genome Hybridization (CGH)}

Genomic DNA isolated from patient and control iPSCs using PureLink Genomic DNA Mini Kit (Life Technologies) was subjected to CNV analysis on 180k Cytosure ISCA v2 arrays. Arrays were designed to be used in two-color experiments i.e., one sample was labeled with fluorescent dye (Cy3) and another sample was labeled with fluorescent dye (Cy5). Array CGH was performed according to the manufacturer's recommendations. Dye swap design was used for DNA labeling and post hybridization and washing, the slide was scanned with an Agilent G2565CA Microarray Scanner. Raw data were generated using the accompanied Feature Extraction software (Agilent Technologies, Santa Clara, CA, USA). Data were analyzed by means of the CytoSure Interpret software v.10 (OGT).

\section{Differentiation of human iPSCs into neurons with a cortical fate}

Differentiation was done as previously described ${ }^{21}$. Induced pluripotent stem cells were dissociated into single cells by using accutase (Sigma-Aldrich) and were plated on matrigel 
(hES qualified matrigel; BD) coated plates/coverslips using mTeSR medium supplemented with $10 \mu \mathrm{M}$ ROCK inhibitor (Calbiochem, Spring Valley, CA, USA). This day was designated as 'day -2 '. Post 48 hours, i.e., on 'day 0', medium was changed to chemically defined default medium (DDM) supplemented with B-27 (Life Technologies; $10 \mathrm{~mL}$ per $500 \mathrm{~mL}$ of medium) and the differentiation was induced by adding $100 \mathrm{ng} / \mathrm{mL}$ of Noggin (R\&D Systems, USA) for 16 days with alternate days of medium change. After the $16^{\text {th }}$ day, cells were cultured by using DDM supplemented with B-27 but without Noggin until day 24. Neural progenitor cells (NPCs) were mechanically dissociated and plated (using DDM with B-27 medium) at a density of 50,000 cells per well of a 12-well plate having coverslips $(18 \mathrm{~mm})$ with poly-lysine and Laminin (both from BD biosciences). ROCK inhibitor $(10 \mu \mathrm{M})$ was added to aid survival. Approximately a week later, half of the medium was replaced with Neurobasal medium (Life Technologies) supplemented with B-27 (10 mL B-27 over $500 \mathrm{~mL}$ medium) along with L-Glutamine $(2 \mathrm{mM})$. This procedure was repeated every $5-7$ days.

\section{Sholl Analysis}

Cells differentiated on coverslips were fixed at day 40 of differentiation using 4\% PFA and immunostained with antibody against anti-MAP2. NIH ImageJ software with built-in 'Sholl analysis' option was used to quantify neuritic complexity. Analysis was done by specifying a centre point at the middle of each soma and by placing a grid with concentric rings of radii increasing in increments of $10 \mu \mathrm{m}$ and the number of intersections was used to estimate the total dendritic branch.

\section{Neuronal connectivity}

Wheat germ agglutinin (WGA) was used as a trans-synaptic tracer to verify neuronal connectivity 22,23 . Three weeks old neurons were transfected with $1 \mu \mathrm{g}$ of MSCV-AcGFPP2A-WGA construct ${ }^{1}$ using Lipofectamin 3000 reagent (Life Technologies) according to the manufacturer's instructions. Seven days after transfection, neurons were fixed with $4 \%$ PFA and performed immunocytochemistry. The primary antibodies were mouse anti- GFP (1:1000; Millipore, Billerica, MA, USA) and rabbit anti-WGA (1:5000; Sigma-Aldrich) antibodies.

\section{Synaptic puncta quantification}

Quantification of co-localized pre- and post-synaptic puncta was performed after immunostaining with Vglut-1 and PSD95 as previously described. The primary antibodies (PSD95, 1:1000, NeuroMab, CA; Vglut-1, 1:1000, Synaptic Systems, Germany; and MAP-2, 1:2000, Sigma-Aldrich) were incubated for $2 \mathrm{~h}$. After post treatment with secondary antibodies and incubation for $1 \mathrm{~h}$, coverslips were mounted and slides were analyzed under a fluorescence microscope (Z1 Axio Observer Apotome, Zeiss). Only Vglut1 and PSD95 puncta along MAP-2 positive processes were counted. Lentivirus overexpression and knockdown of MECP2 were performed as described previously ${ }^{14}$. 


\section{Spine density and morphology}

pHIV7/Syn-EGFP lentivirus was prepared by transfecting HEK cells using the transfection agent polyethylenimine (PEI) (Polysciences, Inc), pSyn-EGFP and packaging plasmids pMDL, Rev RSV and VSVG. During cortical neuron differentiation, on day 40, control and $M E C P 2$ dup neurons were transduced with pHIV7/syn-EGFP. Post transduction, neurons were further differentiated until day 70 and were fixed with $4 \%$ paraformaldehyde and further processed for imaging. Confocal images were obtained using a confocal laserscanning microscope (Nikon), 60x oil objective, with a sequential acquisition setting at $4096 \times 4096$ pixels resolution. Each image is a Z- series projection of approximately 7 to 15 images, averaged two times and taken at $0.5 \mu \mathrm{m}$ depth intervals. GFP labeled differentiated neurons were chosen randomly for quantification. Quantitative analysis was performed blind. Dendrites on primary branches were selected randomly and for each neuron 2-3 segments of $10 \mu \mathrm{m}$ were analyzed. Morphometric measurements were performed manually using the Image $\mathbf{J}$ software (1.44p version). Each spine on the dendrites was manually traced. The length and head width of each spine was automatically measured and used to categorize the spines ${ }^{24}$. Specifically, spines where defined as thin and filopodia (immature) or mushroom and stubby (mature) according to the criteria defined previously ${ }^{24}$. Twentytwo $(51$ segments of $10 \mu \mathrm{m})$ and 18 (42 segments of $10 \mu \mathrm{m})$ neurons were analyzed for the control and MECP2dup neurons, respectively; two clones for each condition.

\section{Multi-electrode array assays (MEA)}

To generate functional neural networks, growing NPCs were dissociated with Accutase and placed on 6 well dishes $\left(10^{6}\right.$ cell per well) under shaker agitation $(95 \mathrm{rpm})$ at $37^{\circ} \mathrm{C}$. The media used was DMEM-F12 supplemented with $0.5 \mathrm{X} \mathrm{B-27,} 0.5 \mathrm{X} \mathrm{N} 2$ and $20 \mathrm{ng} / \mathrm{mL}$ of bFGF (all from Life Technologies). The next day (day 0) neural fate was induced by removing bFGF and adding $10 \mu \mathrm{M}$ Rock inhibitor Y-27632 (Tocris, USA). Two days later (day 2) media was changed to fresh media without Rock inhibitor. The differentiation took place for 2 weeks in suspension, with media change every 4 days. On day 15 of differentiation, these 3D neurospheres cultures were plated on MEA probes containing dual chambers (MED-P5D15A, from Panasonic, Japan) with DMEM-F12 media supplemented with $0.5 \mathrm{X}$ B-27, $0.5 \mathrm{X} \mathrm{N} 2$ and 1\%FBS. Media was changed every 3-4 days. After 2 weeks, media was replaced with Neurobasal supplemented with 1X B-27 and 1:400 Glutamax. Recording of spontaneous spike activity was done using the MED64 System (Panasonic). For Tetrodotoxin (TTX) assays, functional cultures were submitted to TTX treatment $(1 \mu \mathrm{M})$ and spontaneous activity was recorded before, during and $1 \mathrm{~h}$ after washout of TTX. For epigenetic compounds treatment, neurons were treated every other day for 1 week with 10 $\mu \mathrm{M}$ of Scriptaid or NCH-51, Activity was measured in the following week. Only MEA channels with similar number and density of cells plated, and with more than 10 spikes in 5 min interval were used in the analysis. Glutamatergic agonists (AP5 and NBQX) were used to check neuronal activity. After measurement, neurons were immunostained to check morphology and density. Recorded spike was analyzed with the software Neuroexplorer (Nex Technologies, Madison, AL, USA). 


\section{InCell Western and epigenetic compound screening}

Total protein level content was analyzed using the InCell Western technology (LiCor). NPCs were seeded in 96 well plates for imaging $\left(1.4 \times 10^{4}\right.$ cells per well) with DMEM-F12 media supplemented with $0.5 \mathrm{X}$ B-27, $0.5 \mathrm{X} \mathrm{N} 2$ and $20 \mathrm{ng} / \mathrm{mL}$ of bFGF (all from Life Technologies). The next day (day 0 ) neural differentiation was induced by removing bFGF and adding $10 \mu \mathrm{M}$ Rock inhibitor Y-27632 (Tocris). Two days later (day 2) media was changed to fresh media without Rock inhibitor. Media was changed every 3-4 days. After 3 weeks of differentiation, cells were treated in replicates (at least 4 wells per condition) with compounds of the Screen-Well Epigenetics library BML-2836 (Enzo Life Sciences, USA). It comprises 43 compounds carrying activity against lysine modifying enzymes. A single dose of $10 \mu \mathrm{M}$ was used in this screening for 1 week with complete media change every 2 days. On the final day of screening (day 28 of differentiation), cells were fixed with $100 \%$ methanol for $10 \mathrm{~min}$, washed 3 times with PBS and blocked with blocking buffer for fluorescent Western Blotting (Rockland, Pottstown, PA, USA). After 4h of blocking, PSD95 (NeuroMab) primary antibody was added at 1:250 dilution in blocking buffer. Cells were incubated $16 \mathrm{~h}$ at $4{ }^{\circ} \mathrm{C}$ under light agitation. After wash (5 times) with PBS-Tween $0.1 \%$, cells were incubated with 800 IRDye conjugated secondary antibodies (LiCor) at 1:800 dilution in blocking buffer with Tween-20 $0.2 \%$ for $1 \mathrm{~h}$ at room temperature and moderate agitation. For normalization, DRAQ5 and Sapphire700 (LiCor) were added concomitantly with secondary antibody. After incubation, cells were washed (5 times) with PBS-Tween-20 $0.1 \%$ under moderate agitation. Plates were read on Odyssey CLx infrared imaging system (LiCor). PSD95 signal was normalized to cell content (DRAQ5 and Sapphire700) and results were plotted using the software GraphPad Prism.

\section{Statistical Analysis}

At appropriate places, results were expressed as means \pm s.e.m.. Statistical analysis was performed by unpaired Student's $t$ test or one-way analysis of variance (ANOVA), where $P$ $<0.05$ was considered significant and $\mathrm{P}<0.01$ was considered as highly significant.

\section{Results}

\section{Genetic characterization of the MECP2 duplication locus}

Duplication of the $\mathrm{Xq} 28$ chromosomal region, including $M E C P 2$, is a well-known chromosomal rearrangement that is characteristically non-recurrent in nature. Duplication sizes and gene content are highly variable among patients, but genotype-phenotype studies in humans and mice confirmed the increased dosage of $\mathrm{MeCP} 2$ as the underlying cause of the severe neurodevelopmental phenotypes ${ }^{1,2,16-18}$. In this study we included 3 male patients with different Xq28 duplication sizes, ranging from $300 \mathrm{~Kb}$ up to $15 \mathrm{Mb}$ in size, all involving the $M E C P 2$ gene (Figure 1a). Patient 1 duplication is restricted to the $M E C P 2$ gene. Clinical history of the respective patients is described in the Methods section.

\section{Increased MECP2 expression does not affect iPSC derivation}

MECP2dup and control male iPSC clones (see Supplementary Table 1) were derived from fibroblasts using retroviral reprogramming vectors (Sox2, Oct4, c-Myc and Klf4) as 
previously described ${ }^{19}$. The different iPSC lines were validated by analyzing the endogenous expression of pluripotent genes at the transcript level, and secondly by analyzing their potency to generate the three germ layers by embryoid body (EB) in vitro differentiation and in vivo teratoma assays (Supplementary Figure 1a-e). We confirmed by array comparative genomic hybridization (array $\mathrm{CGH}$ ) that the $\mathrm{Xq} 28$ copy number variation $(\mathrm{CNV})$, initially present in the patients' cell lines, remained stable during reprogramming (Supplementary Figure 1f). IPSC lines that accumulated additional CNVs during reprogramming were excluded from the study. Western blot analysis confirmed a two-fold overexpression of $\mathrm{MeCP} 2$ in all reprogrammed patients' cell lines, reflecting the double dosage of MECP2 (Supplementary Figure 1g). Overall, our data demonstrate that increased $\mathrm{MeCP} 2$ dosage does not interfere with the reprogramming process.

\section{Patient and control iPSCs can be differentiated into neuronal cells of cortical identity}

Since cortical dysfunction is observed in patients with MECP2 duplication syndrome, we chose to study the pathophysiology mechanism in iPSC-derived neurons with cortical identity. We used a differentiation protocol that involves an intrinsic mechanism for efficient generation of forebrain progenitors and pyramidal neurons from human iPSCs ${ }^{21}$ (a schematic summary can be seen in the Supplementary Figure 1h). Immunostaining confirmed the neuroectoderm progenitor identity of the cells obtained after 20-30 days of differentiation (Figure 1b). Analysis of transcripts during the early course of differentiation (day 20-30) showed a difference in the expression of $B L B P$, a marker for neural progenitors, and $F O X G 1$, a marker for telencephalic identity, with patient-derived neural progenitor cells (NPCs) expressing more than the control NPCs (Figure 1c and Supplementary Figure 1i for individual clones). SOX1, marker for neural progenitors, also presented a trend of being more expressed in MECP2dup samples (Figure 1b and Supplementary Figure 1i for individual clones). Differentiation of these NPCs resulted in a neuronal population with cortical-like identity that demonstrated higher expression levels of CTIP2 (layer V cortex marker) and TBRI (subplate, Layer I and VI marker) compared to controls (Figure 2a and Supplementary Figure 2a for individual clones). Expression of RELN (Cajal Retzius neurons layer I cortex), however, was significantly reduced in patient neurons compared to control neurons (Figure 2a and Supplementary Figure 2a for individual clones). This result is consistent with previous mouse data, suggesting that MeCP2 negatively regulates the expression of Reelin ${ }^{25}$. Importantly, we observed a high consistency between very different duplication size samples (such as patients 1 and 2), confirming the dominant role of MeCP2 in these phenotypes (Supplementary Figure 2a). RT-qPCR and Western blot analysis confirmed the overall overexpression of $\mathrm{MeCP} 2$ during the whole process of differentiation (Figure $2 b, c$ and Supplementary Figure $2 b$ for individual clones). Finally, immunostaining of mature neurons confirmed the expression of cortical identity markers TBR1 and CTIP2 in neurons derived from affected and controls (Figure 2d).

To determine whether the derived iPSCs are able to generate functional neurons, we used the Multi-Electrode Array (MEA) electrophysiology technique. MEA electrophysiology is a non-invasive way to explore the network activity and connectivity status of neurons ${ }^{26}$. Using an in-house developed protocol to generate three-dimensional neurospheres (detailed in Methods and illustrated in Supplementary Figure 2c-f), we successfully record and 
manipulate actions potentials from our neuronal networks (Figure 2e). Finally, we used a bicistronic lentiviral vector system co-expressing green fluorescent protein (GFP) and wheat germ agglutinin (WGA) for network tracing ${ }^{22,23}$. Although WGA is a non-replicating tracer that undergoes dilution at each synaptic transfer, limiting its quantitative potential, this technique is useful in revealing the presence of physical contacts between iPSC-derived neurons (Figure 2f). No significant difference in the number of WGA positive neurons was found between MECP2dup and controls (Supplementary Figure 2g,h).

\section{Increased glutamatergic synapse number and altered dendritic morphology in MECP2dup neurons}

Previous studies in mice showed that $M E C P 2$ dosage has a major impact on the formation of excitatory synapses. Decreased MeCP2 levels result in a lower number of glutamatergic synapses, while increased MeCP2 dosage leads to increased synaptic numbers ${ }^{27}$. However, more in-depth studies showed that the increased spine density due to increased MeCP2 protein dosage is mainly present in early neurogenesis and decreased with age ${ }^{11}$. Human iPSCs-derived neurons from RTT have a decreased number of glutamatergic synapses ${ }^{14}$, indicating a pivotal role of $\mathrm{MeCP} 2$ in the homeostasis of excitatory synapses. Gene expression profile of neurons derived from patients with $M E C P 2$ duplication syndrome revealed a trend towards a higher level of expression of the synaptic genes VGLUT1 and PSD95 (Figure 2a and Supplementary Figure 2a for individual clones). Moreover, puncta quantification of co-localized pre- and post-synaptic markers confirmed an increased number of glutamatergic synapses in patient derived neurons compared to controls (Figure 3a and Supplementary Figure 3a for individual clones). To confirm the major influence of $\mathrm{MeCP} 2$ dosage in the observed phenotypes, we extended the puncta analysis in control and $M E C P 2$ dup neurons in which the levels of $M E C P 2$ expression were modulated, either by overexpression (in control neurons) or by repression using shRNA (in MECP2dup neurons) (Supplementary Figure 3b,d). Additionally, Western blot analysis confirmed the presence of higher Synapsin-1 and PSD95 protein levels in patient-derived neurons (Figure $3 \mathrm{~b}$ and Supplementary Figure 3c,d).

To study the effect of increased MeCP2 dosage on neuronal morphology, we investigated the dendritic arborization using Sholl analysis. Studies in mice models reported contrasting results, depending on the experimental design ${ }^{11,28,29}$. Our data indicate that human $M E C P 2$ dup neurons have significantly increased dendritic arborization compared to control neurons (Figure 3c). To study spine morphology and density, neurons were transduced with pHIV7/syn-EGFP and further differentiated until day 70, to enable correct morphological analysis. GFP-labeled dendrites were randomly selected and spines were analyzed manually for their morphology, as previously described ${ }^{24}$. As shown in figure $3 \mathrm{~d}$, the total spine density per primary dendritic unit did not differ significantly between control and patient neurons. However, given the significant increased dendritic complexity, the absolute number of spines will be higher in the disease neurons. Interestingly, we observed more immature and less mature spines per dendritic length in the MECP2dup neurons. Alterations of dendritic spines or spine dysgenesis represent a common hallmark of intellectual disability and related synaptopathies ${ }^{30}$. 
A recent paper demonstrated the importance of Cux 1 in regulating dendritic branching and spine density in upper layer neurons ${ }^{31}$. Western blotting confirmed an up-regulation of Cux 1 protein levels in our MECP2 duplication samples compared to controls, indicating that MeCP2 may in fact be regulating the levels of Cux1 in our cultures (Figure $3 \mathrm{e}$ and Supplementary Figure 3e for individual clones). Chromatin immunoprecipitation (ChIP) analysis demonstrated the occupancy of the $\mathrm{MeCP} 2$ protein on the $C U X 1$ promoter region, suggesting a potential downstream target for regulation (Figure $3 \mathrm{f}$ and Supplementary Figure 3f).

\section{Altered network phenotype of MECP2dup neurons}

Although most studies of the nervous system using iPSC rely on the premise that a synaptic phenotype in vitro could ultimately drive a network phenotype, few explored the real implications of these findings. To better explore this venue, we investigated if the imbalance in glutamatergic synapses could drive a functional neuronal phenotype using MEA. Aligned roster plots of independent channels show that both MECP2dup and control neuronal cultures displayed neuronal synchronized activity, measured by overlapping spike burst from different channels (Figure 3g,h and Supplementary Figure 3g,h). Synchronized activity spreading through multiple electrodes indicates information flow in the neuronal network via synapse signalling. Interestingly, overlapping the spike activity histogram from different channels reveals a distinct frequency of synchronized activity for MECP2dup neuronal networks, with significantly more synchronized burst events compared to controls (Figure $3 \mathrm{~g}$ and Supplementary Figure 3h). Again, we could confirm the central role of MeCP2 dosage on the functional phenotype, by repeating the MEA assays in control and $M E C P 2$ dup neurons in which the levels of $M E C P 2$ expression were modulated, as described above (Figure 3i).

\section{Epigenetic modifiers screening in MECP2dup neurons}

Given the multiple roles of MeCP2 at the epigenetic level, we decided to explore the possibility of rescuing the neuronal phenotypes using an epigenetic modifier library of compounds (Screen-Well Epigenetics library from Enzo, see methods for a description and Supplementary Table 2). Using the InCell Western screening technology (ICW), we measured levels of PSD95 as a read out for our screening (Figure 4a) and confirmed that this new tool is able to replicate the results from Western blotting (Figure 3b). This method of screening proves to be reliable and fast, avoiding the demanding human input required in the current state-of-the-art techniques. PSD95 was selected given its core importance in the formation of mature synapses. Additionally, given its physical interactions with several other synaptic proteins, found mutated in many neurodevelopmental syndromes like autism (Supplementary Figure 4a), a screening platform based on this phenotype can be useful to screen therapeutic compounds in these neurodevelopmental disorders as well.

Using a library of 43 compounds (schematic protocol in Supplementary Figure 4b), we found 5 inhibitors that matched two criteria: 1) having a very significant $\mathrm{p}$-value score $(\mathrm{p}<0.001)$, and 2) reducing PSD95 protein levels close to control levels after 1 week of treatment (Figure $4 \mathrm{~b}$ and Supplementary Table 2 and 3). Their mechanisms of action are scattered over distinct categories, for example, histone deacetylase (HDAC) inhibitors 
(Scriptaid, NCH-51 and NSC-3852), DNA methyltransferase inhibitor (Zebularine) and Histone methyltransferase inhibitor (BIX-01294). The HDAC inhibitors Scriptaid and $\mathrm{NCH}-51$ were chosen to further access their potential in rescuing the other observed $M E C P 2$ dup neuronal phenotypes.

First, we measured the impact of these two compounds in rescuing neuronal morphology (Figure $4 \mathrm{c}-\mathrm{d}$ and Supplementary Table 4 for statistics). Given that both inhibitors rescued the branching phenotype to control levels, we then quantified co-localized puncta number after treatment with the epigenetic modifiers (Figure 4e). Once more, both compounds were able to rescue the glutamatergic imbalance present in the MECP2dup neurons. In addition, although these compounds seem to regulate glutamatergic synapse number in $M E C P 2$ dup samples, they did not affect the control neurons (Supplementary Figure 4c-d), suggesting a specific target pathway. Finally, MEA electrophysiology was used to assess whether we could also rescue the altered network phenotype (Figure 4f). MECP2dup neurons presented a higher number of action potentials than the control cultures, which correlates with the higher level of synchronized burst activity (Figure 3g,h and Supplementary Figure 3i). After treatment, $M E C P 2$ dup neurons treated with $\mathrm{NCH}-51$ showed reduced levels of activity, almost to the same level as seen in the controls (Figure 4f). Interestingly, patient neurons treated with Scriptaid had their activity completely abolished.

\section{Discussion}

To study human neurons with altered $M E C P 2$ gene dosage, we derived iPSC from fibroblasts isolated from three independent patients with $M E C P 2$ duplication syndrome. Although one of the patients carries a large duplication of $15 \mathrm{Mb}$, involving many other Xlinked genes, the data obtained from different patients seems to be in coherence in most of the assays, including the MEA electrophysiology. Moreover, the central role of MeCP2 in neuronal connectivity and electrophysiology was further confirmed when its expression was modulated in control or disease neurons, respectively. These experimental data confirm the many clinical observations, indicating the dominant role of the increased MeCP2 dosage in the described phenotypes.

The increased MeCP2 expression did not interfere with the reprogramming and neuronal differentiation processes in our study. Cortical neurons derived from human MECP2dup iPSC lines showed an increased number of glutamatergic synapses when compared to nonaffected controls and RTT derived neurons ${ }^{14}$. We also found increased dendritic arborization and complexity in these neurons, again in sharp contrast to what we observed in RTT derived neurons ${ }^{14}$. This is also in agreement with transgenic mouse data, confirming that $M E C P 2$ overexpression promotes early postnatal dendritic and synaptic growth ${ }^{7,11}$. In addition, we observed altered spine morphology, a hallmark of many neurodevelopmental disorders, and it will be of interest to further investigate this observation in post-mortem brain samples ${ }^{30}$.

MeCP2 was reported to modulate multiple pathways affecting dendritic plasticity ${ }^{12}$. From the myriad of genes that may be affected by MeCP2, we observed increased levels of CUX1, a gene previously identified as a dendritic branching modulator ${ }^{31}$. CUX1 controls the 
development of dendritic branching and promotes dendritic spine development and stabilization during early neuronal differentiation. In a Drosophila model, increasing levels of $\mathrm{Cut}$, the fly homologue, resulted in increased branching and elevated dendritic spike numbers $^{32}$. We speculate that $C U X 1$ expression can be upregulated by direct binding of $\mathrm{MeCP}$, which might constitute a potential mechanism for some of the observed MECP2 syndrome pathophysiology in our human in vitro model.

Electrophysiological analysis using MEA showed that MECP2dup neurons have a significant increased frequency of activity and synchronized bursts compared to controls. In combination with the increased glutamatergic synaptic puncta number observed, it suggests the presence of more active synapses on these neurons, which allows this fast pace flow of information through the network. A similar observation was seen in two different transgenic mouse models, where overexpression resulted in enhanced synaptic plasticity, at least in young animals ${ }^{7,8}$. Our study in human neurons illustrates the importance of correct $M E C P 2$ dosage for synaptic plasticity and homeostasis during brain development and function. Failure of homeostasis, either by haploinsufficiency or overexpression will result in neurodevelopmental phenotypes ${ }^{12}$. To our knowledge, this is the first time that synchronized burst events from iPSCs-derived human neuronal networks are recorded on MEA, without using co-culture with astrocytes ${ }^{33-35}$. Our analysis show a clear correlation between synapse number and functional synchronized activity in human iPSC-derived neurons, revealing the potential of MEA to the study of neurodevelopmental disorders that involve synaptic deficits.

In an attempt to identify compounds that could rescue the effects of increased MeCP2 dosage, we adapted the technology of InCell Western (ICW) to measure the levels of PSD95 protein in our cultures. This synaptic protein was chosen given its core function in synaptic formation and the synaptic imbalance found in $M E C P 2$ dup cultures. Using an epigenetic chemical library, we encountered two HDAC inhibitors that were able to reduce PSD95 protein to control levels, but did not affect control neurons. Treatment of MECP2dup neurons with these two HDAC inhibitors (Scriptaid or NCH-51) for a week was sufficient to rescue several aspects of neuronal morphology. It is known that MeCP2 exerts its gene silencing ability through chromatin modification mediated by its interaction with Sin3A/ HDACI or Ski/NcoR/HDACII repression complexes ${ }^{36}$, pointing this pathway as a potential therapeutic avenue for this syndrome. However, our data also suggest caution on the quest for therapeutic compounds. While both HDAC inhibitors seem to rescue the aberrant morphology, only NCH-51 is able to correctly ameliorate the functional phenotype. Ultimately, this demonstrates the power and necessity of accessing several cellular and functional rescues before in vivo tests. This strategy could reduce downstream failures and expenses in the quest of therapeutic compounds.

Our data indicate that human iPSCs not only can recapitulate some aspects of a genetic neurodevelopmental disorder caused by a genomic duplication, but can also be used in assays to screen potential drugs for the ability to rescue neuronal phenotypes. This cellular model and the novel techniques developed here may help investigators to better design and anticipate results from translational medicine studies, with the potential to lead to the discovery of new compounds to treat neurodevelopmental disorders. 


\section{Supplementary Material}

Refer to Web version on PubMed Central for supplementary material.

\section{Acknowledgments}

This work was supported by grants from the California Institute for Regenerative Medicine (CIRM) TR2-01814 and TR4-06747, the National Institutes of Health through the NIH Director's New Innovator Award Program (1DP2-OD006495-01), R01MH094753, the International Rett Syndrome Foundation (IRSF) and a NARSAD Independent Investigator Grant to ARM. A NINDS K08 NS062711 (MBR), the Fonds voor Wetenschappelijk Onderzoek (FWO) Vlaanderen (G.0767.13) (HVE) and Fondation Jérôme Lejeune, France (SN, HVE). HVE is a senior clinical investigator of the FWO Vlaanderen. EP has been supported by a FWO aspirant fellowship and by a Methusalem grant (KU Leuven and Flanders government). We are grateful to Prof. Nadif-Kasri for sharing a control iPSC cell line and to the patients and their families who participated in this study.

\section{References}

1. Van Esch H, Bauters M, Ignatius J, Jansen M, Raynaud M, Hollanders K, et al. Duplication of the MECP2 region is a frequent cause of severe mental retardation and progressive neurological symptoms in males. Am J Hum Genet. 2005; 77:442-453. [PubMed: 16080119]

2. del Gaudio D, Fang P, Scaglia F, Ward PA, Craigen WJ, Glaze DG, et al. Increased MECP2 gene copy number as the result of genomic duplication in neurodevelopmentally delayed males. Genet Med. 2006; 8:784-792. [PubMed: 17172942]

3. Ramocki MB, Peters SU, Tavyev YJ, Zhang F, Carvalho CM, Schaaf CP, et al. Autism and other neuropsychiatric symptoms are prevalent in individuals with $\mathrm{MeCP} 2$ duplication syndrome. Ann Neurol. 2009; 66:771-782. [PubMed: 20035514]

4. Ramocki MB, Tavyev YJ, Peters SU. The MECP2 duplication syndrome. Am J Med Genet A. 2010; 152A:1079-1088. [PubMed: 20425814]

5. Van Esch H. MECP2 Duplication Syndrome. Mol Syndromol. 2012; 2:128-136. [PubMed: 22679399]

6. Luikenhuis S, Giacometti E, Beard CF, Jaenisch R. Expression of MeCP2 in postmitotic neurons rescues Rett syndrome in mice. Proc Natl Acad Sci U S A. 2004; 101:6033-6038. [PubMed: 15069197]

7. Collins AL, Levenson JM, Vilaythong AP, Richman R, Armstrong DL, Noebels JL, et al. Mild overexpression of MeCP2 causes a progressive neurological disorder in mice. Hum Mol Genet. 2004; 13:2679-2689. [PubMed: 15351775]

8. Na ES, Nelson ED, Adachi M, Autry AE, Mahgoub MA, Kavalali ET, et al. A mouse model for MeCP2 duplication syndrome: MeCP2 overexpression impairs learning and memory and synaptic transmission. J Neurosci. 2012; 32:3109-3117. [PubMed: 22378884]

9. Samaco RC, Mandel-Brehm C, McGraw CM, Shaw CA, McGill BE, Zoghbi HY. Crh and Oprm1 mediate anxiety-related behavior and social approach in a mouse model of MECP2 duplication syndrome. Nat Genet. 2012; 44:206-211. [PubMed: 22231481]

10. Jung BP, Jugloff DG, Zhang G, Logan R, Brown S, Eubanks JH. The expression of methyl CpG binding factor MeCP2 correlates with cellular differentiation in the developing rat brain and in cultured cells. J Neurobiol. 2003; 55:86-96. [PubMed: 12605461]

11. Jiang M, Ash RT, Baker SA, Suter B, Ferguson A, Park J, et al. Dendritic arborization and spine dynamics are abnormal in the mouse model of MECP2 duplication syndrome. J Neurosci. 2013; 33:19518-19533. [PubMed: 24336718]

12. Chahrour M, Zoghbi HY. The story of Rett syndrome: from clinic to neurobiology. Neuron. 2007; 56:422-437. [PubMed: 17988628]

13. Chailangkarn T, Acab A, Muotri AR. Modeling neurodevelopmental disorders using human neurons. Curr Opin Neurobiol. 2012; 22:785-790. [PubMed: 22717528]

14. Marchetto MC, Carromeu C, Acab A, Yu D, Yeo GW, Mu Y, et al. A model for neural development and treatment of Rett syndrome using human induced pluripotent stem cells. Cell. 2010; 143:527-539. [PubMed: 21074045] 
15. Muotri AR, Marchetto MC, Coufal NG, Oefner R, Yeo G, Nakashima K, et al. L1 retrotransposition in neurons is modulated by MeCP2. Nature. 2010; 468:443-446. [PubMed: 21085180]

16. Bauters M, Van Esch H, Friez MJ, Boespflug-Tanguy O, Zenker M, Vianna-Morgante AM, et al. Nonrecurrent MECP2 duplications mediated by genomic architecture-driven DNA breaks and break-induced replication repair. Genome Res. 2008; 18:847-858. [PubMed: 18385275]

17. Carvalho CM, Zhang F, Liu P, Patel A, Sahoo T, Bacino CA, et al. Complex rearrangements in patients with duplications of MECP2 can occur by fork stalling and template switching. Hum Mol Genet. 2009; 18:2188-2203. [PubMed: 19324899]

18. Carvalho CM, Pehlivan D, Ramocki MB, Fang P, Alleva B, Franco LM, et al. Replicative mechanisms for CNV formation are error prone. Nat Genet. 2013; 45:1319-1326. [PubMed: 24056715]

19. Takahashi K, Tanabe K, Ohnuki M, Narita M, Ichisaka T, Tomoda K, et al. Induction of pluripotent stem cells from adult human fibroblasts by defined factors. Cell. 2007; 131:861-872. [PubMed: 18035408]

20. Griesi-Oliveira K, Acab A, Gupta AR, Sunaga DY, Chailangkarn T, Nicol X, et al. Modeling nonsyndromic autism and the impact of TRPC6 disruption in human neurons. Mol Psychiatry. 2014 Epub ahead of print.

21. Espuny-Camacho I, Michelsen KA, Gall D, Linaro D, Hasche A, Bonnefont J, et al. Pyramidal neurons derived from human pluripotent stem cells integrate efficiently into mouse brain circuits in vivo. Neuron. 2013; 77:440-456. [PubMed: 23395372]

22. Ohashi Y, Tsubota T, Sato A, Koyano KW, Tamura K, Miyashita Y. A bicistronic lentiviral vector-based method for differential transsynaptic tracing of neural circuits. Mol Cell Neurosci. 2011; 46:136-47. [PubMed: 20816792]

23. Damak S, Mosinger B, Margolskee RF. Transsynaptic transport of wheat germ agglutinin expressed in a subset of type II taste cells of transgenic mice. BMC Neurosci. 2008; 9:96. [PubMed: 18831764]

24. Harris KM, Jensen FE, Tsao B. Three-dimensional structure of dendritic spines and synapses in rat hippocampus (CA1) at postnatal day 15 and adult ages: implications for the maturation of synaptic physiology and long-term potentiation. J Neurosci. 1992; 12:2685-2705. [PubMed: 1613552]

25. Kundakovic M, Chen Y, Guidotti A, Grayson DR. The reelin and GAD67 promoters are activated by epigenetic drugs that facilitate the disruption of local repressor complexes. Mol Pharmacol. 2009; 75:342-354. [PubMed: 19029285]

26. Spira ME, Hai A. Multi-electrode array technologies for neuroscience and cardiology. Nat Nanotechnol. 2013; 8:83-94. [PubMed: 23380931]

27. Chao HT, Zoghbi HY, Rosenmund C. MeCP2 controls excitatory synaptic strength by regulating glutamatergic synapse number. Neuron. 2007; 56:58-65. [PubMed: 17920015]

28. Jugloff DG, Jung BP, Purushotham D, Logan R, Eubanks JH. Increased dendritic complexity and axonal length in cultured mouse cortical neurons overexpressing methyl-CpG-binding protein MeCP2. Neurobiol Dis. 2005; 19:18-27. [PubMed: 15837557]

29. Zhou Z, Hong EJ, Cohen S, Zhao WN, Ho HY, Schmidt L, et al. Brain-specific phosphorylation of $\mathrm{MeCP} 2$ regulates activity-dependent Bdnf transcription, dendritic growth, and spine maturation. Neuron. 2006; 52:255-269. [PubMed: 17046689]

30. Sala C, Segal M. Dendritic spines: the locus of structural and functional plasticity. Physiol Rev. 2014; 94:141-88. [PubMed: 24382885]

31. Cubelos B, Sebastián-Serrano A, Beccari L, Calcagnotto ME, Cisneros E, Kim S, et al. Cux1 and Cux2 regulate dendritic branching, spine morphology, and synapses of the upper layer neurons of the cortex. Neuron. 2010; 66:523-535. [PubMed: 20510857]

32. Grueber WB, Jan LY, Jan YN. Different levels of the homeodomain protein cut regulate distinct dendrite branching patterns of Drosophila multidendritic neurons. Cell. 2003; 112:805-818. [PubMed: 12654247]

33. Li Y, Wang H, Muffat J, Cheng AW, Orlando DA, Lovén J, et al. Global transcriptional and translational repression in human-embryonic-stem-cell-derived Rett syndrome neurons. Cell Stem Cell. 2013; 13:446-58. [PubMed: 24094325] 
34. Wainger BJ, Kiskinis E, Mellin C, Wiskow O, Han SS, Sandoe J, et al. Intrinsic membrane hyperexcitability of amyotrophic lateral sclerosis patient-derived motor neurons. Cell Rep. 2014; 7:1-11. [PubMed: 24703839]

35. Odawara A, Saitoh Y, Alhebshi AH, Gotoh M, Suzuki I. Long-term electrophysiological activity and pharmacological response of a human induced pluripotent stem cell-derived neuron and astrocyte co-culture. Biochem Biophys Res Commun. 2014; 443:1176-81. [PubMed: 24406164]

36. Nan X, Ng HH, Johnson CA, Laherty CD, Turner BM, Eisenman RN, et al. Transcriptional repression by the methyl-CpG-binding protein MeCP2 involves a histone deacetylase complex. Nature. 1998; 393:386-389. [PubMed: 9620804] 


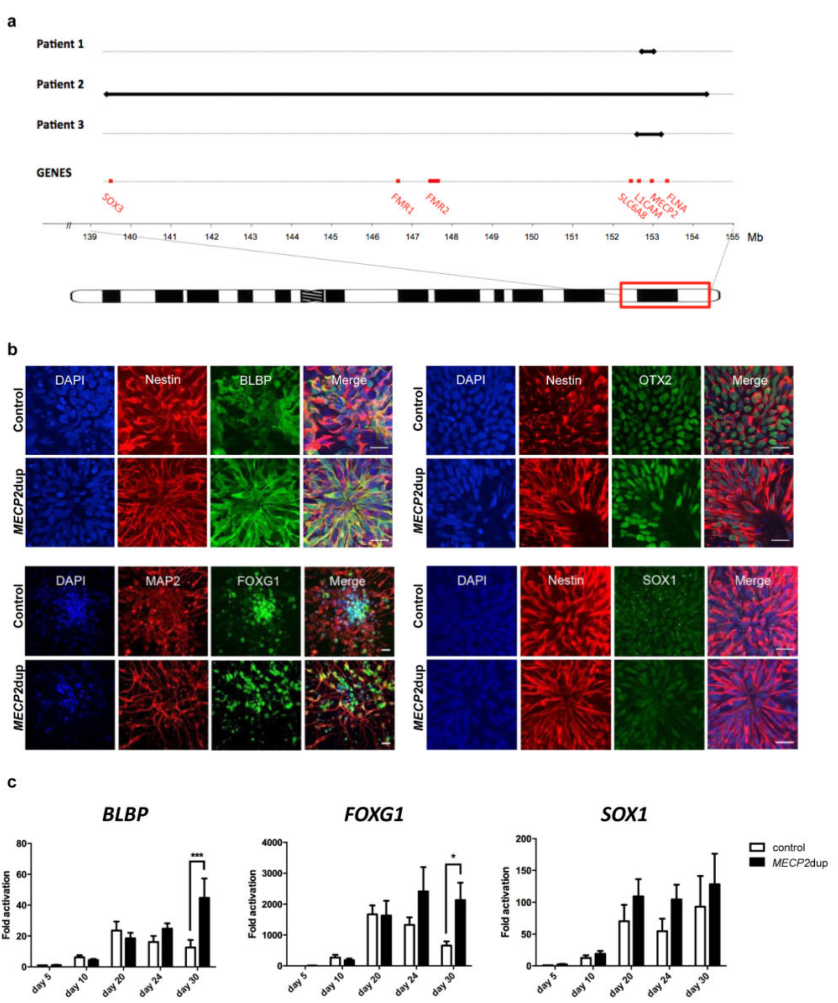

Figure 1. Altered expression of neural progenitor genes in NPCs derived from MECP2dup iPSCs a) Schematic representation of the duplication sizes for each patient (black line). The location of the MECP2 gene as well as other genes known to be involved in X-linked intellectual disability are shown (based on NCBI36/hg18).

b) Immunofluorescence images of control and MECP2dup NPCs showing the expression of neural progenitor genes co-stained with either Nestin (day 20) or MAP-2 (day 30). Scale bar represents $20 \mu \mathrm{m}$.

c) Transcript analysis of neural progenitor genes on control and MECP2dup NPCs by qRTPCR during the course of differentiation (day 5 to day 30). Graphs show the average of three different biological replicates and are represented as fold difference compared to 'day 5' of control. Data is representative of mean \pm s.e.m., $n>3$ independent differentiation experiments ( $\mathrm{t}$ test, $* * * \mathrm{p}<0.001, * \mathrm{p}<0.05)$. 


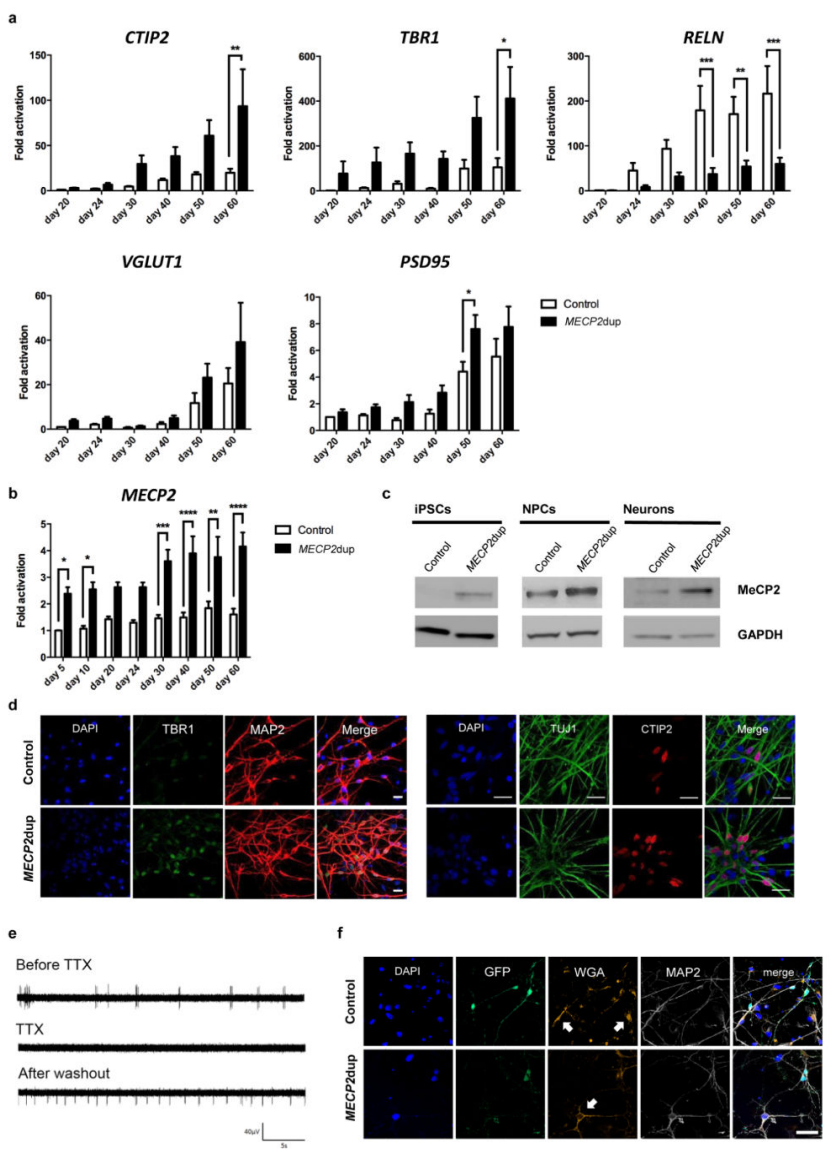

Figure 2. $M E C P 2 d u p$ derived neurons exhibit modulation in cortical gene expression a) Control and $M E C P 2$ dup neurons were analyzed for the expression of cortical and synaptic genes over the course of differentiation by qRT-PCR. Data is represented as fold difference compared to 'day 20' of control cells. Data is representative of mean \pm s.e.m., $n>3$ independent differentiation experiments ( $\mathrm{t}$ test, $* * * \mathrm{p}<0.001, * * \mathrm{p}<0.01, * \mathrm{p}<0.05$ ).

b) Expression by qRT-PCR of MECP2 in control and MECP2dup cells over the course of differentiation towards neurons. Data is representative of mean \pm s.e.m., $n>3$ independent differentiation experiments ( $\mathrm{t}$ test, $* * * \mathrm{p}<0.001$, **p $<0.01, * \mathrm{p}<0.05$ ).

c) Western blotting analysis of MeCP2 protein in iPSCs, NPCs and neurons from control and $M E C P 2$ dup samples. Protein extracts were prepared at days 0 (iPSCs), 20 (NPCs) and 60 (Neurons). GAPDH is shown as a protein loading control.

d) Representative immunostaining images are shown of control and MECP2dup neurons at day 40 of differentiation showing the increased expression of cortical proteins. Cells are costained with either MAP2 or TUJ1. Scale bar represents $20 \mu \mathrm{m}$.

e) Spontaneous neuronal activity and its inhibition by TTX treatment of cells plated on multi-electrode arrays (MEA), demonstrating the biological nature of the signal detected. The figure shows one representative channel of a control sample, before, during and after washout of TTX treatment. 
f) WGA experiment showing the proximity of neuronal connections in our neuronal network. White arrows point the cells to which WGA spread. Cells transduced with the WGA vector are shown in green (GFP reporter). Scale bar represents $40 \mu \mathrm{m}$. 
a

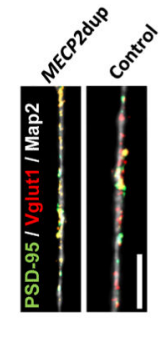

c

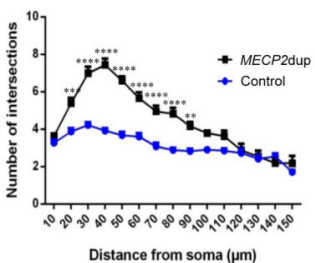

Distance from soma ( $\mu \mathrm{m})$
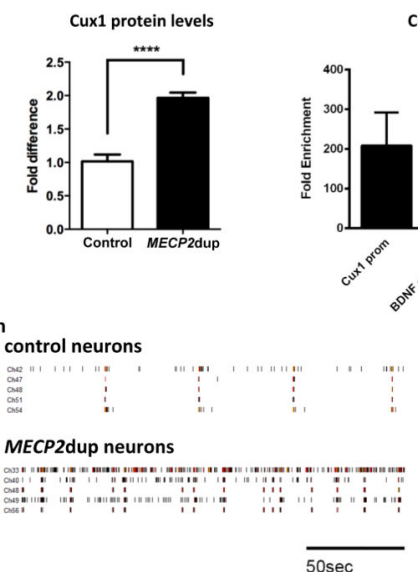

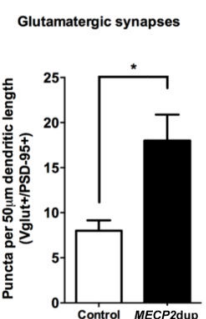

d

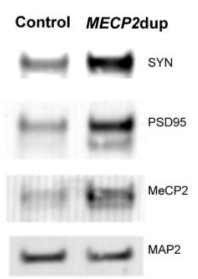

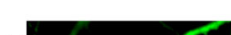

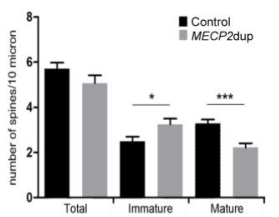

g

ChIP MeCP2

Sync burst in 5 min intervals
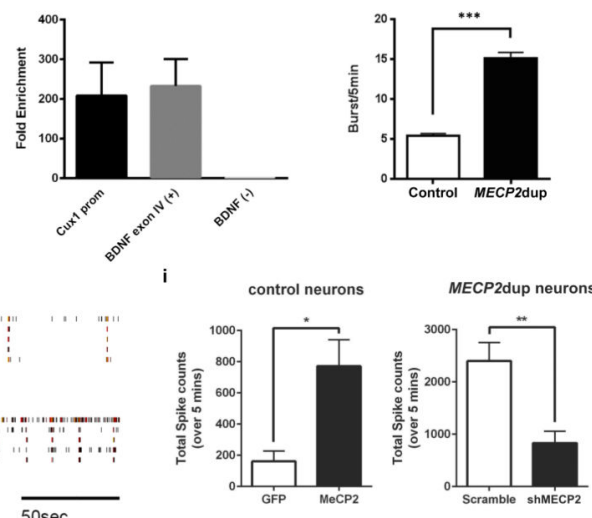

Figure 3. MECP2dup cortical neurons exhibit increased synaptic gene expression and dendritic arborization

a) Puncta quantification of post- and pre-synaptic markers. The synaptic proteins VGlut1 (pre-synaptic) and PSD95 (post-synaptic) were used as markers and only co-localized puncta were quantified and graphed. Data is representative of mean \pm s.e.m., $n=3$ independent differentiation experiments, ${ }^{*} \mathrm{p}=0.0324$, two-tailed t-test. Scale bar represents $10 \mu \mathrm{m}$.

b) Western blotting analysis of synaptic proteins, synapsin and PSD95 as well as MeCP2 in total cell lysates of day 50 cortical neurons. MAP2 is used as a protein loading control.

c) Sholl analysis of control and $M E C P 2$ dup cortical neurons. Data is representative of mean \pm s.e.m., $n>3$ independent differentiation experiments. For statistical significance, 125 control neurons and $80 \mathrm{MECP} 2$ dup neurons were considered for analysis ( $\mathrm{t}$ test, ${ }^{* * *} \mathrm{p}<$ $0.001, * * \mathrm{p}<0.01, * \mathrm{p}<0.05)$.

d) Left panel: representative high magnification confocal images of GFP-labeled neurons at day 70 in vitro from control and $M E C P 2$ dup neurons. Scale bar represents $5 \mu \mathrm{m}$; Right panel: quantification of the total spine density and density of the mature and immature spines per dendritic section. Histograms represent the mean values \pm s.e.m., $* \mathrm{p}=0.02$, **** $\mathrm{p}=0.0003$, Mann Whitney test. 
e) Western blotting analysis of Cux1 protein levels in MECP2dup NPCs compared to controls. Protein levels were quantified by Odyssey using 2 control cells lines and 3 $M E C P 2$ dup cell lines. Data is representative of mean \pm s.e.m., $\mathrm{n}=3$ biological replicates for each cell line. Unpaired $\mathrm{t}$ test was used for statistics $(* * * \mathrm{p}<0.0001)$.

f) Association of MeCP2 with $C U X 1$ promoter, $B D N F$ exon 4 (positive control) or $B D N F$ negative control was determined by ChIP assays with anti-MeCP2 antibody. Chromatin was isolated from 4 weeks $M E C P 2$ dup neurons. Fold enrichment was quantified by qPCR.

Histograms represent the mean \pm s.e.m. Three biological with three or four technical repeats each.

g) Quantification of the total number of synchronized bursts per 5 min interval present in at least 4 different channels. Data is representative of mean \pm s.e.m. $n=3$ independent differentiation experiments. Unpaired t-test, $* * * * p<0.0001$.

h) Aligned raster plots of 5 representative channels for control and MECP2dup neurons on MEA. Spontaneous activity is shown for a total period of $200 \mathrm{~s}$.

i) Functional phenotype after overexpression and knockdown of MECP2 in control and $M E C P 2$ dup neurons, respectively. Total spikes counts are shown, 1 week after virus transduction. GFP: overexpression control virus; $M E C P 2: M E C P 2$ overexpression virus; scramble: shRNA scramble virus; shMECP2: shRNA for $M E C P 2$ transcript. Unpaired t test used $\left(* \mathrm{p}=0.012,{ }^{*} \mathrm{p}=0.0028\right)$. Bars represent mean \pm s.e.m. Three independent differentiation experiments. 
a

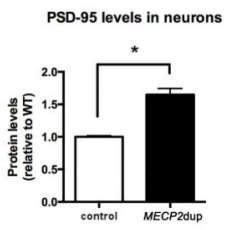

b
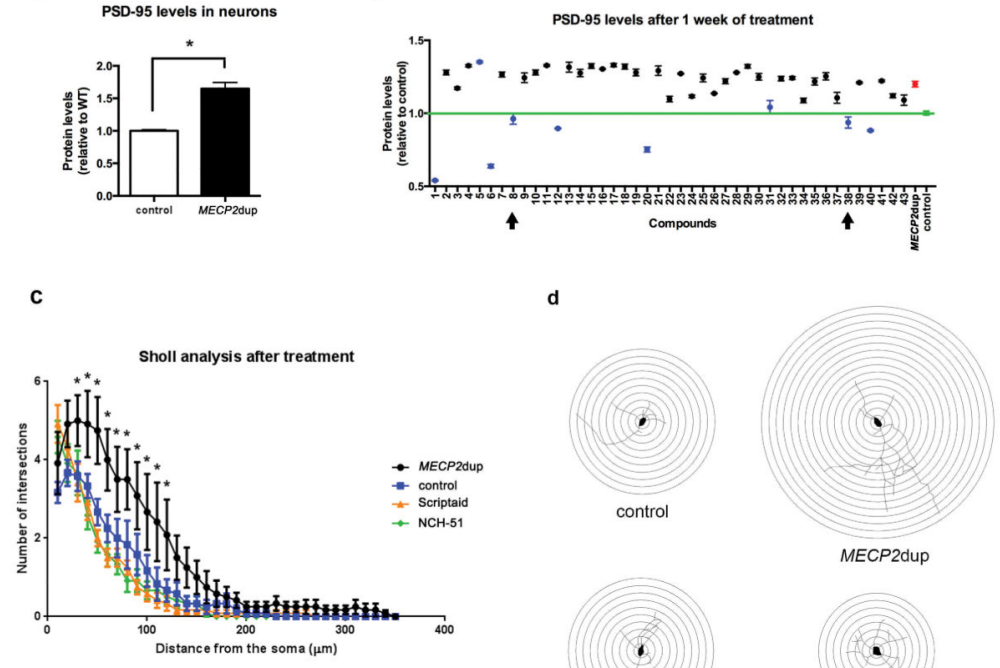

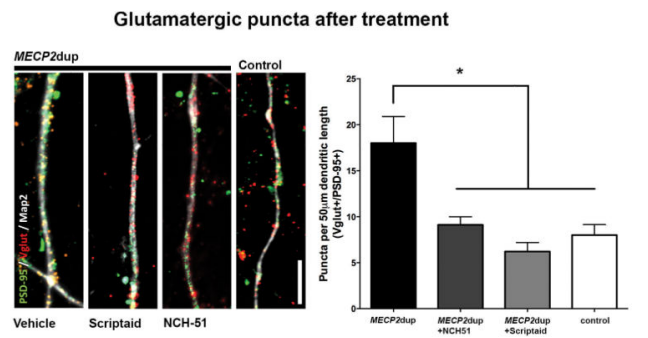

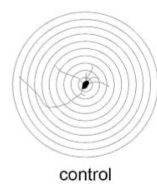

MECP2dup
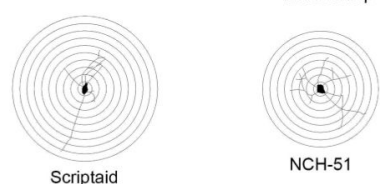

$\overline{50 \mathrm{~mm}}$

f

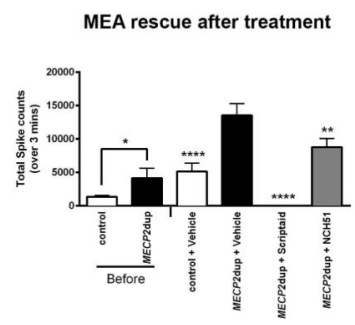

Figure 4. Electrophysiology network properties and morphological rescue of $\mathrm{MeCP2dup}$ neurons a) InCell Western (ICW) in 50 days differentiated neurons for PSD95 reveals an upregulation in patient cells. Protein levels are shown relative to controls. Data is representative of mean \pm s.e.m., $n=3$ independent differentiation experiments. Unpaired ttest, $* \mathrm{p}=0.0146$.

b) Compound library screening in 50 days differentiated neurons. Screen-Well Epigenetics library (Enzo) was used. The neurons were treated for one week before ICW. Green, control neurons PSD95 levels; Red, MECP2dup neurons PSD95 levels; Blue, treated neurons that had a p value $<0.0001$ when compared to untreated duplicated neurons. Control neurons had a p value $<0.0001$ when compared to untreated MECP2dup neurons. One-way ANOVA test used and bars represent \pm s.e.m. Arrows indicate the compounds Scriptaid (compound 8) and $\mathrm{NCH}-51$ (compound 38), selected to further investigation.

c) Sholl analysis of control and patient neurons that were treated for 1 week with the indicated compounds. Data is representative of mean \pm s.e.m., 3 independent differentiations experiments, 24 neurons counted on each condition $(* \mathrm{p}<0.05)$.

d) Sholl analysis diagrams of representative neurons. Concentric circles have a distance of $10 \mu \mathrm{m}$ between them.

e) Puncta quantification of post- and pre-synaptic markers after treatment with epigenetic modifiers. The synaptic proteins Vglut1 (pre-synaptic) and PSD95 (post-synaptic) were used 
as markers and only co-localized puncta were quantified and graphed. Scale bar represents $10 \mu \mathrm{m}$. One-way ANOVA test used to represent data after treatment using Dunnett's multiple comparison test and comparing to $M E C P 2 \operatorname{dup}(* \mathrm{p}<0.05$ ). $\mathrm{p}$ values per comparison: $M E C P 2$ dup $v s$ control $=\mathrm{p}<0.05 ; M E C P 2$ dup $v s$ MECP2dup $+\mathrm{NCH}-51=\mathrm{p}<$ 0.01; MECP2dup vs MECP2dup+Scriptaid = $\mathrm{p}<0.0001$. Bars represent mean \pm s.e.m.

Three independent differentiation experiments.

f) Functional rescue after treatment with epigenetic modifiers; MEA was used to measure activity. Total spikes counts are shown before and after treatment with compounds.

Unpaired $\mathrm{t}$ test used to compare control and MECP2dup data before treatment $(* \mathrm{p}=0.0110)$. One-way ANOVA test used to represent data after treatment using Dunnett's multiple comparison test comparing to MECP2dup+Vehicle (**p < 0.01, ****p < 0.0001). Bars represent mean \pm s.e.m. Three independent differentiation experiments. 